\title{
The influence of multiple temporal memories in the peak-interval procedure
}

\author{
A. George Wilson • Matthew S. Matell • \\ Jonathon D. Crystal
}

Published online: 3 March 2015

(C) Psychonomic Society, Inc. 2015

\begin{abstract}
Memories for when an event has occurred are used to anticipate future occurrences of the event, but what happens when the event is equally likely to occur at two different times? In this study, one group of rats was always reinforced at $21 \mathrm{~s}$ on the peak-interval procedure (21-only group), whereas another group of rats was reinforced at either 8 or $21 \mathrm{~s}$, which varied daily (8-21 group). At the beginning of each session, the behavior of the 8-21 group largely lacked temporal control, but by the end of the session, temporal control was reestablished. When both groups were reinforced at $21 \mathrm{~s}$, the patterns of responding were indistinguishable after subjects in the 8-21 group had experienced 13 reinforcement trials. Finally, the reinforcement times of previous sessions affected the 8-21 group, such that subjects were biased depending on the reinforcement time of the prior session. These results show that when the reinforcement time is initially ambiguous, rats respond in a way that combines their expectations of both possibilities; then they incrementally adjust their responding as they receive more information, but still information from prior sessions biases their initial expectation for the reinforcement time. Combined, these results imply that rats are sensitive to the age of encoded temporal memories in an
\end{abstract}

A. G. Wilson

Department of Psychology, University of Kentucky, Lexington, KY, USA

A. G. Wilson · J. D. Crystal $(\bowtie)$

Department of Psychological and Brain Sciences, Indiana University, Bloomington, IN 47405-7007, USA

e-mail: jcrystal@indiana.edu

A. G. Wilson • J. D. Crystal

Department of Psychology, University of Georgia, Athens, GA, USA

M. S. Matell

Department of Psychology, Villanova University, Villanova, PA, USA environment in which the reinforcement time is variable. How these results inform the scalar expectancy theory, the currently accepted model of interval-timing behavior, is discussed.

Keywords Temporal memory $\cdot$ Temporal control $\cdot$ Interval timing $\cdot$ Peak-interval procedure $\cdot$ Multiple memories $\cdot$ Rat

To track the passage of time, organisms employ at least three physiologically distinctive mechanisms that are each separately affected by several physiological (Wearden, Philpott, \& Win, 1999) and pharmacological (Meck, 1996) factors. Which mechanism is used to complete a task is dependent on the length of time that must be monitored. Two of these timing mechanisms, millisecond timing (employed when subsecond durations must be monitored) and circadian timing (employed when durations close to $24 \mathrm{~h}$ must be monitored), have high precision but are limited to a small range in the durations that may be accurately timed, and they may be slow to resynchronize to a change in environmental conditions. In contrast, interval timing is used to track a wide range of durations lasting seconds or minutes, and this mechanism can be rapidly adjusted to respond to changing environmental conditions, but as a consequence it is highly variable (Buhusi \& Meck, 2005). Despite the flexibility of interval-timing processes, the predominant theory of interval timing, the scalar expectancy theory (Gibbon, 1977; Gibbon, Church, \& Meck, 1984), focuses on well-learned or steady-state performance. This focus may be due to the fact that investigating temporally governed behavior (especially in nonhuman animals) requires one to first capture evidence of temporal control. Temporal control is defined here as behavior that has been shaped to the reinforcement time of the current session. Experimentally inducing a loss of temporal control after a subject has learned a 
temporal relationship would also cause the experimenter to lose, temporarily, access to the very behavior that he or she is attempting to study.

The scalar expectancy theory posits that temporally governed behavior is the result of three interconnected but separate components: the clock, memory, and comparison components (Gibbon, 1977; Gibbon et al., 1984). The clock component marks the currently elapsing passage of time by semirandomly emitting pulses. The accumulator, also part of the clock component, tracks the number of pulses that occur between the initiation of a stimulus and the delivery of a reward. The memory component stores, in reference memory, the total number of pulses registered in the accumulator at the time of reward. Finally, the comparison component is responsible for a decision process that assesses the relative difference between an accumulating number of pulses and a value sampled from memory; when the relative difference between these two values passes below a threshold, the animal begins to respond, but then ceases to respond once the absolute value of this difference exceeds the threshold again.

The scalar expectancy theory is often used to explain the behavior of individual rodents performing the peak-interval procedure. The peak-interval procedure engages rodent subjects in two trial types that occur randomly throughout a session: (1) fixed-interval (FI) trials, on which subjects can depress a response lever after a specific delay has elapsed to earn a reinforcer, and (2) peak-interval (PI) trials on which reinforcement is withheld, resulting in the subject increasing its response rate around the expected time of reinforcement, but then decreasing the response rate when the elapsed interval of time sufficiently has exceeded this expectation. Although the scalar expectancy theory models the cognitive processes that subjects utilize on individual trials of the peak-interval procedure, performance on a single trial is highly variable and does not necessarily reflect temporal control (Gibbon \& Church, 1990; Gibbon et al., 1984). As a result, measures of a subject's expectation of the reinforcement time are often extracted from the average of all PI trials that occur in a session. The high variability on individual PI trials stems from two potential sources of noise in the model of scalar expectancy theory: (1) Pulses emitted by the pacemaker are not always registered by the accumulator (Gibbon et al., 1984), and (2) the temporal memory used by the memory component is randomly selected from a pool of all stored memories of the task (Gibbon \& Church, 1990).

This second source of variability implies that a recently formed memory of the reinforcement time exerts no greater influence on expectation than do memories formed many sessions ago. On the contrary, memories from a current session should have, on the basis of probability, less influence on current behavior than the much larger pool of memories from numerous prior sessions. Discerning the influences of newer memories (from the current session) versus older memories (from prior sessions) on temporally governed behavior can be investigated experimentally by changing the delay to reinforcement, since these two sources of information (i.e., older and newer memories) would then predict different temporal performance. However, such a manipulation would induce a loss of temporal control, and as mentioned above, scalar expectancy theory has yet to be extended to explain such behavior.

Some prior studies have attempted to investigate instances in which temporal control is disrupted due to the time of reinforcement being changed. These prior studies have shown that, in these semivariable environments (e.g., environments in which the time of reinforcement is stable for a period of time, but then changes to a new stable reinforcement time), subjects abruptly shift from using older memories (of the previous reinforcement duration) to newer memories (of the new reinforcement duration) to guide temporally governed behavior.

In Simen, Balci, Cohen, and Holmes (2011), human participants engaged in a time-based "beat-the-clock" task in which a square was displayed on a computer screen for a set amount of time during individual trials and random numbers were overlaid to dissuade participants from counting. During individual trials, participants earned larger amounts of money (a max of $\$ 0.25$ per trial) the closer their first response was to the time that the square disappeared, but a response that occurred after the square had disappeared was not rewarded. The time that the square was displayed (mean $=8 \mathrm{~s}$ ) was constant for only a few trials, and unsignaled changes in the interval ( $>50 \%$ of the current duration) occurred rapidly over the course of the session. The average correlation between the participant's response and the actual deadline exceeded an $R^{2}$ of .9 after only a single trial since an interval change had occurred, meaning that human participants could rapidly adjust their responding as soon as a discrepancy was detected: This could not have been done had all prior memories had equal chances of influencing the subject's expectation.

Similarly, in three rodent studies using the peak-interval procedure, subjects changed their temporal behavior within a session when (1) they were retrained with a new duration (Meck, Komeily-Zadeh, \& Church, 1984) or (2) they experienced a sequence of changing reinforcement times over the course of several sessions (Lejeune, Ferrara, Simons, \& Wearden, 1997); this abrupt change in responding made it unlikely that rodent subjects were equally likely to sample from older versus newly formed (i.e., within-session) memories. However, the transition from a shorter to a longer duration produced a different pattern of responding than did a transition from a longer to a shorter duration (Higa, 1997). This may have been due to the fact that on long sessions, subjects experienced the absence of reinforcement at the short time prior to being reinforced at the long time, whereas on short sessions, reinforcement trials ended before the longer reinforcement durations had been experienced. In either case, if recently formed memories and memories formed earlier 
were equally likely to be sampled, the transitioning behaviors of these rats in all three of the above-mentioned studies should have been gradual.

The studies above suggest that subjects can regain temporal control quickly after detecting a change in the reinforcement time when in a variable environment. Humans were able to use a single presentation of a duration to guide their behavior while ignoring all preceding trials, whereas rodent subjects are capable of transitioning to a new reinforcement time relatively quickly, as well. However, a number of questions still remain about how, specifically, subjects reestablish temporal control in a variable environment. First, what is the least amount of information that is needed before a subject will reestablish temporal control; for example, would it be possible to reestablish temporal control after experiencing a single reinforcement trial? Second, if the current reinforcement time is shorter than the subject's initial expectation, would temporal control be reestablished faster than when the reinforcement time is longer than the initial expectation? Finally, would subjects always use prior experiences to guide behavior? In other words, if the reinforcement time of a current session were selected at random, would the reinforcement time of the preceding session still affect behavior? Answering these questions would not only inform current understanding of an underresearched topic in the interval-timing literature, but it might also provide avenues along which scalar expectancy theory could be updated to better explain how temporal control is reestablished in a variable environment; given that interval timing has been shown to be more sensitive to environmental change than other timing processes (Buhusi \& Meck, 2005), such an addition would improve the external validity of this model.

In the present study, we investigated these questions using a group of rats (the 8-21 group) trained to expect food availability at either of two durations (i.e., either 8 or $21 \mathrm{~s}$ after the start of a trial), and a second group (the 21-only group) that could only earn food at a single duration (i.e., $21 \mathrm{~s}$ after the start of a trial). For the subjects in the 8-21 group, the duration used was randomly selected at the start of each daily session but did not change over the course of the session. In this paradigm, the subjects in the 8-21 group started the session in an initially ambiguous state (i.e., they could not fully predict the reinforcement time), characterized by a lack of temporal control, but developed accurate temporal control after experiencing several reinforcement trials during the session.

\section{Method}

Subjects

Sprague-Dawley rats were restricted to $15 \mathrm{~g}$ of food per day throughout the experiment, but had ad libitum access to water. A 12:12 reverse light:dark cycle was used. Sessions occurred in the dark phase of the cycle, with lights turning off at 7 am and turning on at $7 \mathrm{pm}$. Twenty rats were separated into two groups with the rats being tested in two shifts (counterbalanced across subjects). Shortly after this separation, however, one rodent from the 8-21 group had to be euthanized due to health problems. The first shift was started at approximately 9:00 am and the second at approximately 11:00 am. The rats were trained 5 days per week. All procedures were approved by the Institutional Animal Care and Use Committee at the University of Georgia and followed the guidelines of the National Research Council Guide for the Care and Use of Laboratory Animals.

\section{Materials}

Ten stainless steel operant chambers $(30.5 \times 24.1 \times 29.2 \mathrm{~cm}$; Med Associates), each encased in a sound-attenuating cabinet $(66 \times 35.6 \times 55.9 \mathrm{~cm})$, were used. A feeder dispensed $45-\mathrm{mg}$ dustless grain pellets (Bio-Serv F0165) into a food trough, and a photobeam tracked when the rat's head entered the trough. A retractable lever that the rat could depress when extended was also in the chamber, along with a small hole that allowed a sipper tube from a water bottle to be inserted into the chamber; subjects had free access to water at all times. The experimental procedure was programmed using the MED-PC IV (St. Albans, VT, USA) software package on two computers, both of which were housed in a separate room.

\section{Procedure}

Rats received food magazine directed training for one day, followed by two days of training in which food could be earned by depressing a response lever located to the left of the food magazine on a fixed-ratio 1 schedule. Rats were then trained on the peak-interval procedure. At the onset of a trial, the lever was extended and a white noise was presented until either the subject earned a pellet or the trial ended. In this procedure, a subject received equal numbers (i.e., $50 \%$ ) of randomly selected trial types, either FI or PI trials. During FI trials, the subject had the opportunity to respond and earn one pellet of food after the white-noise stimulus had been on for a specific amount of time. The first response after the interval had elapsed produced the pellet and caused the signal to be terminated and the lever retracted. During PI trials, reinforcement was omitted, and the white noise remained on for a randomly selected time that was between four and five times longer than the FI schedule used in the current session (in 8-s sessions, between 32 and $40 \mathrm{~s}$; in 21-s sessions, between 84 and $105 \mathrm{~s}$ ), at which point the lever was retracted and the noise ended. After either trial type, an intertrial interval (in the absence of noise) occurred for a randomly selected interval between 60 and $100 \mathrm{~s}$. 
For the subjects in the 21-only group, the reinforcement duration of FI trials was always $21 \mathrm{~s}$. In contrast, the 8-21 group subjects received two different reinforcement durations. On randomly selected days the FI was either 8 or $21 \mathrm{~s}$, and the reinforcement duration was held constant within a session. For randomization, a list containing equal numbers of the session types was created and a random number was assigned to each item; the list of random numbers (and the corresponding items) was then sorted in ascending order. To maintain equivalent training, the subjects in the 21-only group either completed a session in which they were reinforced at $21 \mathrm{~s}$ or remained in their home cage and were not run. The same randomization procedure described above was used to select the reinforcement time that the subjects in the 8-21 group experienced within a session, thereby equating across groups the numbers of sessions in which reinforcement occurred at $21 \mathrm{~s}$. Two hours after the session ended, a supplement of food was given to the animals; the supplement plus the food received during the daily session equaled $15 \mathrm{~g}$ of food.

\section{Data analysis}

The data from Sessions 31 to 130 were pooled to create the final data set. The PI trials for a session were fit with a Gaussianlinear model that was initially developed by Buhusi and Meck (2000), with the parameters of the model being estimated using the error-correcting Marquardt-Levenburg algorithm (Marquardt, 1963). Measures of peak time and the coefficient of variation were derived from the Gaussian-linear model using MATLAB (Mathworks, Natick, MA).

On individual trials, a rat's lever-pressing behavior conforms to either a break-run-break or a square-wave pattern (Gibbon \& Church, 1990). The transition between the first break and the run is termed the start time (denoting the start of a high rate of responding), and the transition between the end of the run and the second break is termed the stop time. The start and stop times from individual PI trials were assessed using the methods described in Church, Meck, and Gibbon (1994), in which all possible start and end times were considered and the combination that minimized the error between the observed and expected rates was selected.

The data were grouped by the number of preceding FI trials that had been experienced within the session. Put another way, all PI trials that occurred before any FI trials were grouped together and called "OFI" trials, whereas all PI trials that occurred after a single FI trial had been reinforced were grouped together and called "1FI" trials. Two separate analyses were completed on this generated data set. First, the PI data were further split according to whether the prior session had used the same or a different reinforcement time, to capture whether subjects biased their responding on the basis of the reinforcement time of the prior session. Second, an inflection point analysis was conducted on the start and stop times of all sessions for the 8-21 subjects to quantify the point at which they transitioned from being initially uncertain about the reinforcement time at the beginning of the session to expressing stable temporal control. In this inflection point analysis, one line was fit to the data points from those PI trials that occurred prior to reinforcement (i.e., "OFI" trials) and those that occurred before a specified number of FI trials had occurred; slope and intercept were free parameters. A second model was then fit as a flat line (i.e., slope $=0$ ), with an intercept equal to the average of all remaining data points, ranging from the same number of FIs used in the first model to the last data point. This two-model analysis was then iteratively completed on all possible FIs experienced, and the residual sum of squares (RSS) for each model was calculated; the optimal inflection point (our operational definition of the number of FIs experienced when subjects first expressed stable temporal control) was then taken from the model set with the lowest RSS value. All trials that occurred before temporal control was reestablished were removed, and this subset of data was then submitted to the same Gaussian-linear model fit described above, to determine the peak time and the coefficient of variation.

\section{Results}

\section{Peak-interval response distributions}

The 8-21 subjects and the 21-only subjects had equivalent experiences at the 21-s duration; the only difference between these two groups was that one group had also experienced sessions in which reinforcement could be earned by responding $8 \mathrm{~s}$ after the onset of the same signal. The presence of this additional memory caused the distribution of responses of the 8-21 subjects to be earlier and broader than that of the 21-only subjects (Fig. 1a) on 21-s sessions. During these sessions, the mean peak time of the 8-21 subjects was $17.62 \pm$ $1.09 \mathrm{~s}$ (mean $\pm S E M$ ), and the peak time for the 21-only subjects was $19.65 \pm 1.32 \mathrm{~s}$; the coefficient of variation (i.e., the relative breadth of the response distribution after being normalized by the peak time) of the 8-21 subjects was $0.62 \pm$ 0.05 , whereas the coefficient of variation for the 21-only subjects was $0.49 \pm 0.06$. Between-subjects $t$ tests revealed that significant differences between these two groups were observed in both the peak time $[t(17)=-3.57, p<.05]$ and the coefficient of variation $[t(17)=4.95, p<.05$; see Fig. $1 \mathrm{~b}]$. Furthermore, when the 8-21 rats' peak functions from the 8and 21-s sessions were compared, within-subjects $t$ tests revealed that the coefficient of variation from the short $(8-\mathrm{s})$ sessions $(0.53 \pm 0.06)$ was significantly narrower than that from the long (21-s) sessions $(0.62 \pm 0.05)[t(8)=-5.06, p<$ .05 ; see Fig. 1b]. Finally, the peak time of the $8-21$ subjects on short sessions $(8.51 \pm 0.87)$ was significantly earlier than the 
A Averaged Whole Session Responding

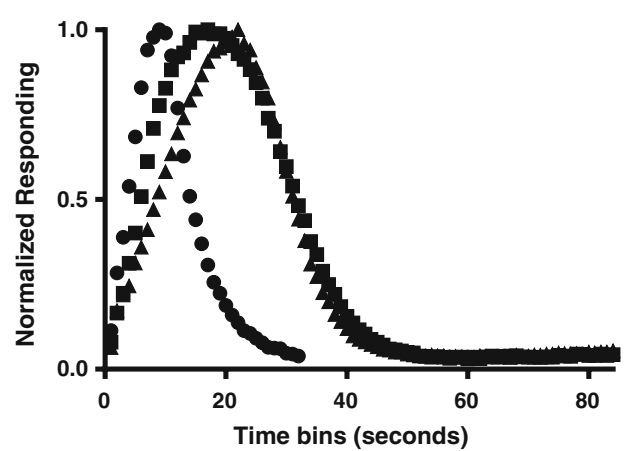

C

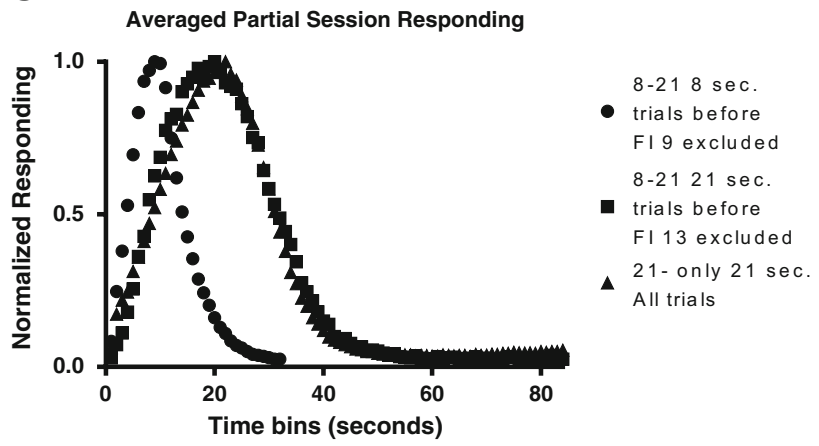

Fig. 1 a Normalized response rates ( $y$-axis) for the entire data set of the 821 subjects and the 21 -only subjects as a function of trial duration ( $x$-axis). b The same data, with relative times (actual time/averaged peak time) on the $x$ axis. A two-model fit was used to find the number of reinforcement trials that had to be experienced before temporal control was reestablished among

peak time obtained when these subjects experienced long sessions $(17.62 \pm 1.09)[t(8)=-37.50, p<.05$; see Fig. 1a], demonstrating that $8-21$ subjects did indeed respond sooner on short than on long sessions.

Individual trials analysis: 8-21 versus 21-only group

As is shown in Fig. 2a (bottom lines), start times differed between the two groups at the beginning of a session but converged after several trials. These visual impressions were confirmed by a $2 \times 21$ mixed-effects analysis of variance (ANOVA) that considered both group and experience (i.e., the number of preceding FI trials), respectively. The ANOVA revealed a significant main effect of experience $[F(20,340)=11.19, p<.05]$, no effect of group $[F(1,17)=$ $0.74, p=.40]$, but a Group $\times$ Experience interaction $[F(20$, $340)=2.43, p<.05]$. Probing the interaction with betweensubjects post-hoc $t$ tests performed on each experience level (i.e., $0 \mathrm{FI}, 1 \mathrm{FI}$, etc.) revealed that only the $0 \mathrm{FI}$ and $1 \mathrm{FI}$ experience levels were significantly different, meaning that the start times for the two groups converged after one reinforcement trial had been experienced. For stop times (Fig. 2a, top lines), the main effect of experience $[F(20,340)=1.97, p<.05]$ and the Group $\times$ Experience interaction $[F(20,340)=7.77, p<.05]$
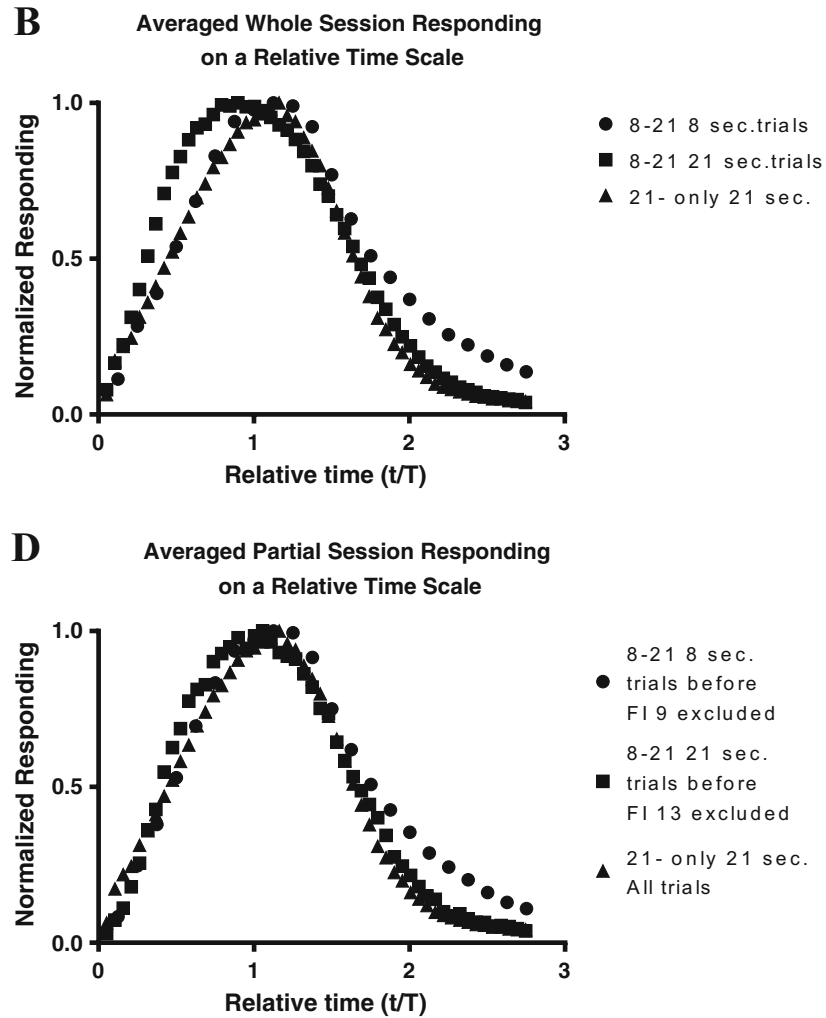

the subjects in the 8-21 group on their 8-s and 21-s sessions. $\mathbf{c}$ and $\mathbf{d}$ Peak trials that occurred before these points were excluded, so that only the data (normalized response rate) from trials occurring after these time points were compared to those of the 21-only group, as a function of (c) real time ( $x$-axis) and (d) relative time ( $x$-axis)

were significant, whereas the main effect of group $[F(1,17)=$ $0.30, p=.59]$ was nonsignificant. Post-hoc, between-subjects $t$ tests performed on this interaction revealed that the stop times in the two groups converged after two reinforcement trials. The effect seen in the stop times followed the same general pattern as the start times: The patterns of responding in these two groups converged after they had experienced only one or two reinforcement trials, as measured by start or stop times, respectively.

Individual-trials analysis: 8-21 group (8- vs. 21-s sessions)

The start and stop times for the 8-21 group on 8- and 21-s sessions are plotted as a function of experience in a session in Fig. 2b. As can be seen, early in a session, subjects began (Fig. 2b, bottom lines) and terminated (Fig. 2b, top lines) responding at roughly the same time. However, as they experienced feedback about the current session's reinforced duration, their start and stop times progressively diverged. To quantify the 8-21 group's responding on the 8- and 21-s sessions, start and stop times were analyzed separately, and $2 \times 21$ within-subjects ANOVAs that considered both reinforcement duration (either 8 or $21 \mathrm{~s}$ ) and experience were conducted. For start times, the main effects of duration $[F(1,8)=108.68, p<.05]$ and 

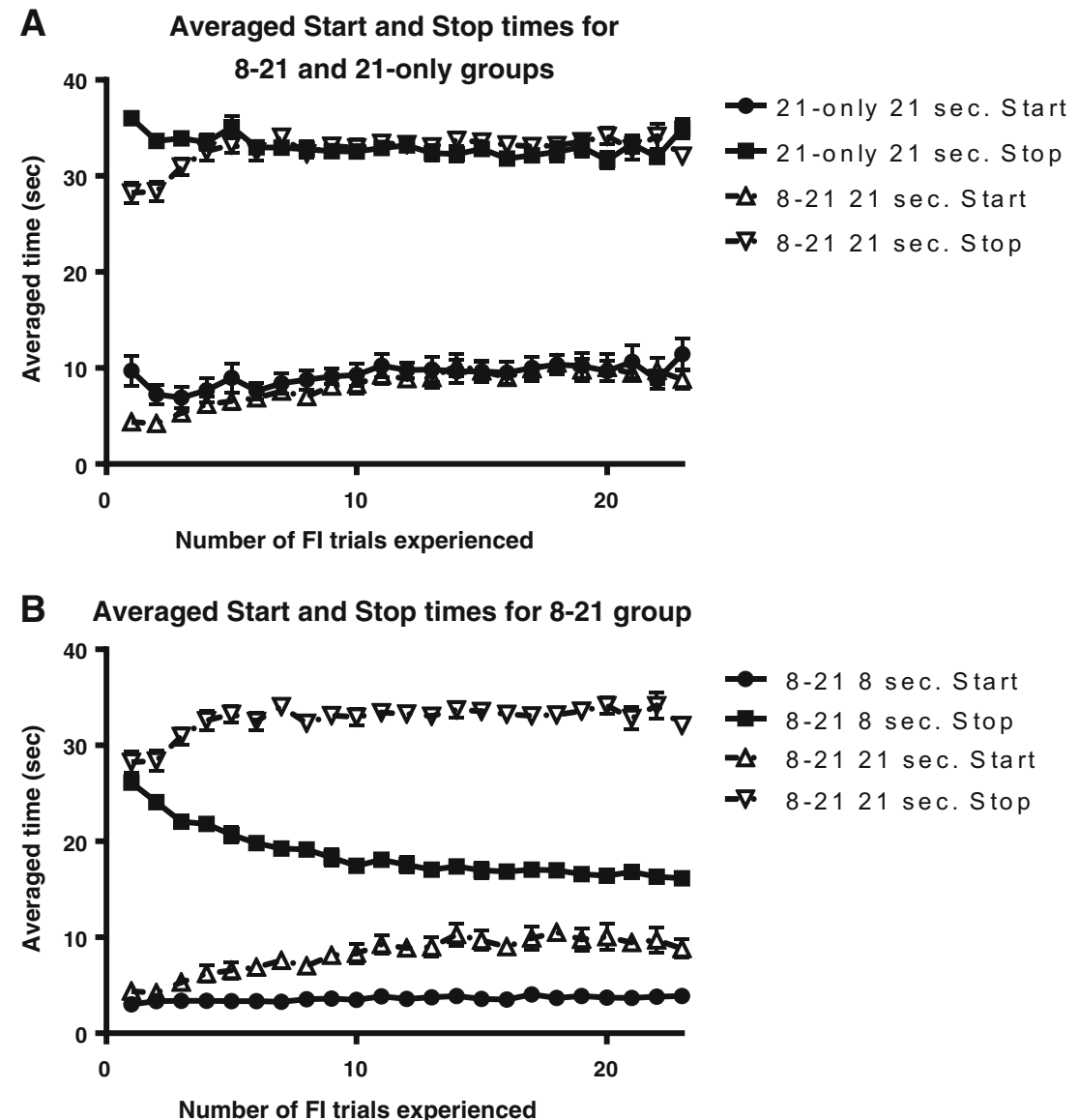

Fig. 2 a The lever-pressing behavior of the 8-21 subjects was different from that of the 21-only subjects early in the sessions; after they had experienced several FI trials, the behavior of these two groups overlapped. Error bars represent the standard errors of the

experience $[F(20,160)=9.37, p<.05]$, as well as the Duration $\times$ Experience interaction $[F(1,8)=9.58, p<.05]$, were significant. Probing the interaction with a post-hoc, within-subjects $t$ test on all experience levels (i.e., $0 \mathrm{FI}, 1 \mathrm{FI}$, etc.) revealed that the start times diverged after a single FI trial. Likewise for stop times, the main effects of duration $[F(1,8)=1132.02, p<$ $.05]$, experience $[F(20,160)=4.53, p<.05]$, and their interaction $[F(20,160)=31.57, p<.05]$ were significant (Fig. 2b, top lines). Post-hoc, within-subjects $t$ tests performed on all experience levels revealed that the stop times also diverged after a single FI trial. Similar to the individual-trial analysis comparing the 21-only and 8-21 groups, the behavior of the 8-21 subjects diverged after a small number of reinforcement trials - in this case, one reinforcement trial, as measured by both start and stop times.

Effect of the previous session on performance in the 8-21 subjects

The data from the $8-21$ subjects were sorted according to whether the current session's reinforcement time was the same means. b The lever-pressing behavior of the 8-21 subjects was indistinguishable early in the session when they were reinforced at either 8 or $21 \mathrm{~s}$, but it diverged after they had experienced a few FI trials

or different from that in the previous session. When rats were reinforced at $8 \mathrm{~s}$ on the current session (see Fig. 3a), a 2 (same vs. different reinforcement time in the prior session) $\times 21$ (experience) within-subjects ANOVA on start times yielded a significant main effect of same versus different $[F(1,8)=$ $6.74, p<.05$ ], with responding beginning later if the previous session had been reinforced at $21 \mathrm{~s}$; a nonsignificant main effect of experience $[F(20,160)=1.08, p=.37]$; and a nonsignificant interaction [Same vs. Different $\times$ FI Experience: $F(20,160)=1.37, p=.14]$. However, a 2 (same vs. different) $\times 21$ (experience) within-subjects ANOVA on the stop times revealed significant main effects of both same versus different $[F(1,8)=14.5, p<.05]$ and experience $[F(20,160)=63.42, p$ $<.05]$, as well as a significant interaction $[F(20,160)=2.04, p$ $<.05]$. Probing the interaction with post-hoc, withinsubjects $t$ tests revealed significant differences at every level of experience prior to receiving 16 reinforcement trials, with the exception of trials preceded by four, six, nine, and ten FI trials. These data demonstrate that the reinforcement time of the previous session caused a difference in both the subject's start and stop times on the 

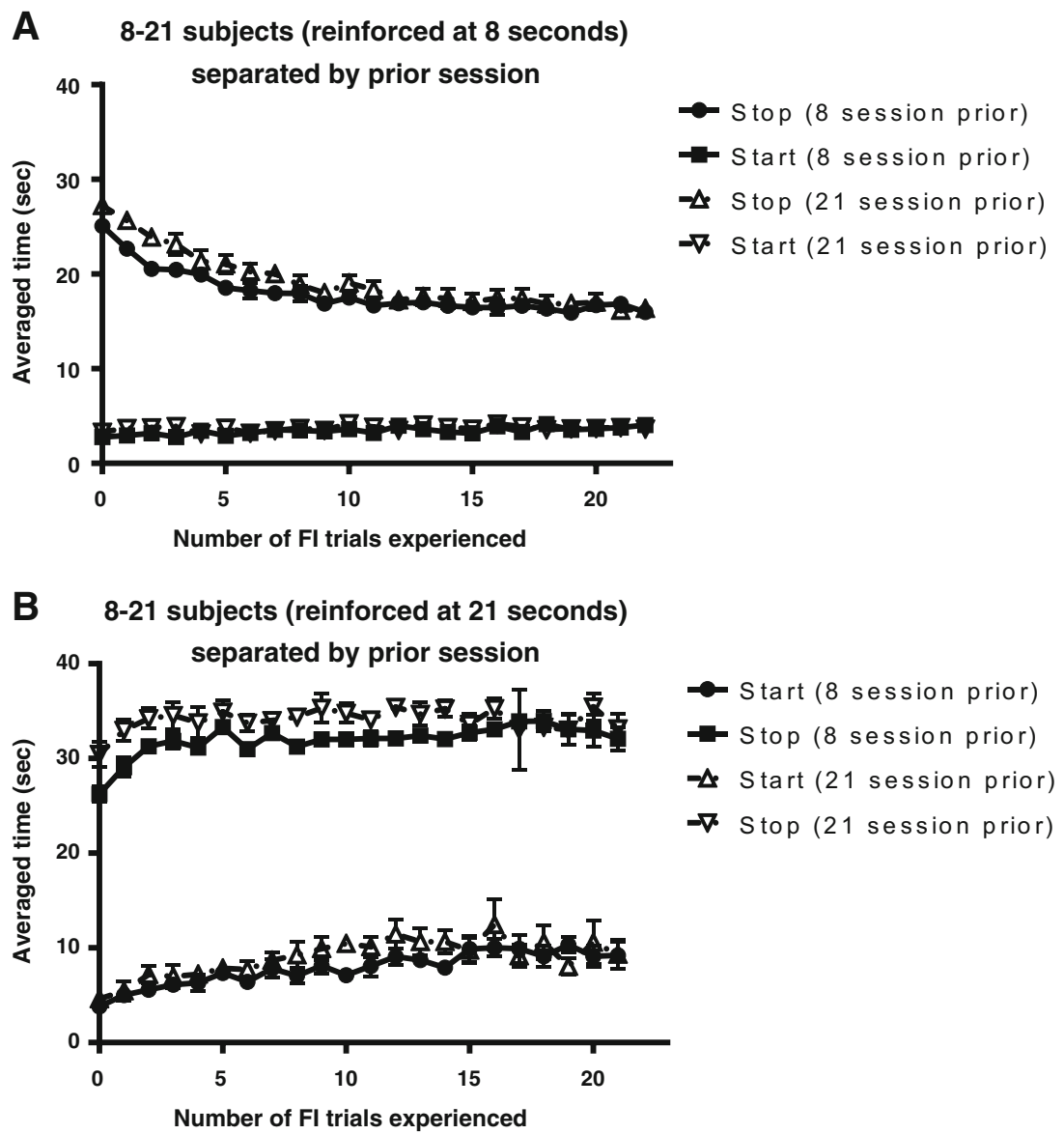

Fig. 3 a The lever-pressing behavior of the 8-21 subjects when they were reinforced at $8 \mathrm{~s}$ was dependent on the previous session; error bars represent the standard errors of the means. If they had been reinforced at $21 \mathrm{~s}$, they began the current session biased toward this time, and if they had been reinforced at $8 \mathrm{~s}$, they began the current session biased toward $8 \mathrm{~s}$. b Like their behavior on days on which the reinforcement time was $8 \mathrm{~s}$, the leverpressing behavior of the $8-21$ subjects when they were reinforced at $21 \mathrm{~s}$ was dependent on the previous session. If they had been reinforced at $21 \mathrm{~s}$, they began the current session biased toward this time, and if they had been reinforced at $8 \mathrm{~s}$, they began the current session biased toward $8 \mathrm{~s}$ current session. The finding of a significant difference in start and stop times due to the reinforcement time of the prior session documents that memories formed approximately $24 \mathrm{~h}$ in the past affect the behavior of the subject on the current session.

On 21-s sessions (see Fig. 3b), single-trial responding was earlier when the previous session had been reinforced at $8 \mathrm{~s}$ than when it had been reinforced at $21 \mathrm{~s}$. A 2 (same vs. different) $\times 21$ (experience) within-subjects ANOVA on start times revealed that the main effects of both same versus different $[F(1,8)=18.36, p<.05]$ and experience $[F(20,160)=$ $9.20, p<.05]$ were significant, but the interaction was nonsignificant $[F(20,160)=1.3, p=.18]$. For stop times, the main effects of same versus different $[F(1,8)=14.74, p<.05]$ and experience $[F(20,160)=4.49, p<.05]$ were also significant, but the interaction was nonsignificant [Same vs. Different $x$ Experience: $F(20,160)=0.96, p=.51]$. As with the data from the 8-s sessions, we can conclude that memories formed over $24 \mathrm{~h}$ earlier biased current behavior.
Inflection point analysis

The start and stop times from the 8-21 subjects, when reinforced at $8 \mathrm{~s}$ and grouped by the number of FI trials that had been experienced, stabilized (as determined by the inflection point) after nine and eight FI trials, respectively. When analyzed in a similar manner, the start and stop times of the 8-21 subject reinforced at $21 \mathrm{~s}$ stabilized after 13 and three FI trials had occurred, respectively. All PI trials that occurred before temporal control had been reestablished (i.e., PI trials that occurred before the 9th FI trial on 8-s sessions, and all PI trials that occurred before the 13th FI trial on 21-s sessions) were removed, and the new peak times and coefficients of variation for this subset of the data were, for the 8-s session, peak time $7.99 \pm 0.29 \mathrm{~s}$ and coefficient of variation $0.69 \pm 0.05$ (means \pm $S E M \mathrm{~s}$ ); for the 21-s session, peak time $18.9 \pm 0.38 \mathrm{~s}$ and coefficient of variation $0.54 \pm 0.02$ (means $\pm S E M \mathrm{~s}$; see Fig. 1c). In comparing the 21 -only group to the $8-21$ subjects when they were under temporal control and reinforced at $21 \mathrm{~s}$, 
no significant difference was apparent in the peak times $[t(17)$ $=-1.27, p=.22]$ or the coefficients of variation $[t(17)=-1.90$, $p=.074$; see Fig. 1d]. This finding suggests that after experiencing several trials within a long session, memories from prior (i.e., short) sessions did not influence the behavior of the subjects in the 8-21 group. Finally, we observed significant differences between the 21-only group and the 8-21 subjects when they were reinforced at $8 \mathrm{~s}$ (and temporal control had been reestablished) in both peak times $[t(17)=-22.5$, $p<.05]$ and the coefficients of variation $[t(17)=-3.84, p<$ $.05]$, meaning that on short sessions, when temporal control had been reestablished in the 8-21 group, the relative breadth of responding was still greater in this group than in the 21-only control group.

\section{Discussion}

Two groups of rats were trained to expect food for responding after an auditory signal had been played for a specific amount of time. One group, the 21-only group, was only reinforced after the signal had been played for $21 \mathrm{~s}$. The other group, the 8-21 group, could earn food after either 8 or $21 \mathrm{~s}$ had elapsed, but during a session only one of these durations was reinforced. We have three main observations from the results. First, the behavior of subjects trained to expect reinforcement at one of two durations (8-21 group) was initially different from that of another group of rats that were trained to time only one of these durations (21-only group), but these two groups eventually expressed similar responding after the subjects in the 8-21 group had experienced multiple (i.e., approximately 13) reinforcement trials. Since the reinforcement time of the current session was randomly selected and constant within a session, rats could have used the first reinforcement trial to determine whether the reinforcement time of the current session was 8 or $21 \mathrm{~s}$ (and had this happened, there would have been only a marginal difference between the 21 -only and $8-21$ subjects when they were reinforced at $21 \mathrm{~s}$ ). However the 8-21 rats appeared to need several, redundant, reinforcement trials before temporal control was reestablished. Second, the development of accurate temporal control in the 8-21 group was dependent on whether the reinforcement time occurred at either 8 or $21 \mathrm{~s}$. Third, and finally, memories from the session just prior affected the current-session performance, since there was a significant difference between the data sets composed of sessions in which the prior session had either the same or a different reinforcement time from the current session. This means that despite the reinforcement time of the current session being selected at random, subjects were sensitive to the reinforcement time of the last session.

Our results suggest that rats may be sensitive to the age of temporal memories, because newer memories appear to have a greater impact on their temporally governed behavior. We note that, although we provided random variation in the age of memories from prior sessions, we did not experimentally manipulate age in a systematic fashion. The currently predominant theory of interval timing, the scalar expectancy theory, does not specify a role for the age of temporal memories or the process(es) by which a subject may transition from uncertainty to certainty about the reinforcement time. As we noted in the introduction, relatively few studies in the interval-timing literature have investigated this topic. However, several studies within the matching-law literature have experimentally disrupted the reinforcement ratio between two available reinforcement schedules in mid-training, to track how behavioral control is reestablished (Kyonka, 2008; Kyonka \& Grace, 2007, 2010). Indeed, the concatenated generalized matching law (Davison \& McCarthy, 1988) states that the degrees to which trials with different reinforcement ratios from prior experiences change behavior are independent and additive; this assertion resonates with the behavior of the subjects in the 821 group because (1) behavior on PI trials changed as a function of the number of preceding FI trials, and (2) the reinforcement time of the prior session affected performance on the current session.

Likewise, a number of cognitive models of human memory have proposed that the age of a memory plays a large role in the impact it will have on a subject's behavior (Bjork \& Whitten, 1974; Brown, Neath, \& Chater, 2007; Rubin \& Wenzel, 1996). Indeed, without such a mechanism one would expect estimates corresponding to both 8 and $21 \mathrm{~s}$ to be drawn throughout the session, since both criterion times had been reinforced many more times in the past, relative to the number received in the current session. Although our study suggests that older memories exert less of an influence on a rat's expectation as more memories from the current session are formed, we cannot conclude that the memories are forgotten or that a memory distribution has only a finite capacity. In fact, it would be difficult to account for the gradual change in temporally governed behavior observed in the subjects if older memories had no influence.

The present findings suggest that with a slight modification to the scalar expectancy theory, specifically to the memory component of the model, these data and previous, similar findings could be modeled by this theory. First, the idea that the memories associated with a single cue are retrieved at random should be revised, since the results here show that rats are sensitive to the age of a temporal memory. Second, a mechanism that allows subjects to selectively use a truncated set of memories to inform behavior - specifically, a mechanism through which newly formed memories exert greater influence than memories from prior sessions - could be added to the memory component of the scalar expectancy theory and potentially account for the reestablishment of temporal control. Although memories from prior sessions did indeed play a role in the behavior captured in this study, the $\sim 20$ 
reinforcement trials from the prior session did not appear to influence the subject's behavior as much as a single reinforcement trial from the current session, as evidenced by the relatively large changes in responding that occur between $0 \mathrm{FI}$ (when there were no memories of a current-session reinforcement trial) and 1FI (when a single memory from the current session was present) in Figs. 2a and b. Conversely, during "same" sessions (i.e., when the reinforcement time of the prior session was the same as that of the current session), the other duration had not been reinforced for at least $48 \mathrm{~h}$, yet the subject's initial pattern of responding was clearly influenced by memories from these prior sessions (see Figs. 3a and b). The exact mechanism for how prior-session memories inform this initial pattern of responding would be better informed by a replication of the present study in which the number of prior sessions on which the reinforcement time was either the same as or different from the reinforcement time of the current session was experimentally controlled.

Any replication of the present study should be cognizant, however, that the difference between the durations used in this experiment (a 2.63 ratio of difference) may have impacted the results. In Experiment 2 of Cheng, Westwood, and Crystal (1993), pigeons were reinforced at one of two durations for pecking the same response key (randomly chosen on each trial) and were presented with test trials in which reinforcement was omitted. Under these conditions, separate peaks of responding occurred when the ratio of the difference between the two durations was large (specifically, the larger duration was five times longer than the shorter duration), but only a single peak of responding that overlapped with both durations occurred when the ratio of difference was small (the long duration was 2.33 times longer than the shorter duration). If the present study were to be replicated using two durations that had a greater ratio of difference, and that were therefore more easily discriminable by the subjects, temporal control might be reestablished sooner.

The details necessary for the scalar expectancy theory to be amended so as to account for the reestablishment of temporal control are not fully captured in this study. The only necessary change to scalar expectancy theory identified on the basis of the present data is that the temporal memories used by the memory component are not chosen at random, and newer memories exert a greater influence on subjects' expectations than do older memories. However, future investigations that would lead to the identification of these parameters should be a priority for researchers in this field. Interval-timing ability has been identified as a mechanism that is more sensitive to environmental change than are other timing processes (Buhusi \& Meck, 2005). With slight modification, scalar expectancy theory has the potential to be able to model this important behavior, and such a change would increase the external validity of this important and widely accepted theoretical construct.
Author note This work was supported by National Institute of Mental Health Grant No. R01MH080052 to J.D.C.

\section{References}

Bjork, R. A., \& Whitten, W. B. (1974). Recency-sensitive retrieval processes in long-term free recall. Cognitive Psychology, 6, 173-189. doi:10.1016/0010-0285(74)90009-7

Brown, G. D. A., Neath, I., \& Chater, N. (2007). A temporal ratio model of memory. Psychological Review, 114, 539-576. doi:10.1037/ 0033-295x.114.3.539

Buhusi, C. V., \& Meck, W. H. (2000). Timing for the absence of a stimulus: The gap paradigm reversed. Journal of Experimental Psychology-Animal Behavior Processes, 26, 305-322. doi:10. 1037/0097-7403.26.3.305

Buhusi, C. V., \& Meck, W. H. (2005). What makes us tick? Functional and neural mechanisms of interval timing. Nature Reviews Neuroscience, 6, 755-765. doi:10.1038/ Nrn1764

Cheng, K., Westwood, R., \& Crystal, J. D. (1993). Memory variance in the peak procedure of timing in pigeons. Journal of Experimental Psychology: Animal Behavior Processes, 19, 68-76. doi:10.1037/ 0097-7403.19.1.68

Church, R. M., Meck, W. H., \& Gibbon, J. (1994). Application of scalar timing theory to individual trials. Journal of Experimental Psychology: Animal Behavior Processes, 20, 135-155. doi:10. 1037/0097-7403.20.2.135

Davison, M., \& McCarthy, D. (1988). The matching law: A research review. Hillsdale, NJ: Erlbaum.

Gibbon, J. (1977). Scalar expectancy theory and Weber's law in animal timing. Psychological Review, 84, 279-325. doi:10.1037/0033295X.84.3.279

Gibbon, J., \& Church, R. M. (1990). Representation of time. Cognition, 37, 23-54. doi:10.1016/0010-0277(90)90017-E

Gibbon, J., Church, R. M., \& Meck, W. H. (1984). Scalar timing in memory. Annals of the New York Academy of Sciences, $423,52-77$.

Higa, J. J. (1997). Dynamics of temporal control in rats: The effects of a brief transition in interval duration. Behavioural Processes, 40, 223 229. doi:10.1016/S0376-6357(97)00021-1

Kyonka, E. G. E. (2008). The matching law and effects of reinforcer rate and magnitude on choice in transition. Behavioural Processes, 78, 210-216. doi:10.1016/J.Beproc.2007.12.003

Kyonka, E. G. E., \& Grace, R. C. (2007). Rapid acquisition of choice and timing in pigeons. Journal of Experimental Psychology: Animal Behavior Processes, 33, 392-408. doi: 10.1037/0097-7403.33.4.392

Kyonka, E. G. E., \& Grace, R. C. (2010). Rapid acquisition of choice and timing and the provenance of the terminal-link effect. Journal of the Experimental Analysis of Behavior, 94, 209-225. doi:10.1901/Jeab. 2010.94-209

Lejeune, H., Ferrara, A., Simons, F., \& Wearden, J. H. (1997). Adjusting to changes in the time of reinforcement: Peak interval transitions in rats. Journal of Experimental Psychology: Animal Behavior Processes, 23, 211-231. doi:10.1037/00977403.23.2.211

Marquardt, D. W. (1963). An algorithm for least-squares estimation of nonlinear parameters. Journal of the Society for Industrial and Applied Mathematics, 11, 431-441.

Meck, W. H. (1996). Neuropharmacology of timing and time perception. Cognitive Brain Research, 3, 227-242. doi:10.1016/0926-6410(96) 00009-2 
Meck, W. H., Komeily-Zadeh, F. N., \& Church, R. M. (1984). Two-step acquisition: Modification of an internal clock's criterion. Journal of Experimental Psychology: Animal Behavior Processes, 10, 297306. doi:10.1037/0097-7403.10.3.297

Rubin, D. C., \& Wenzel, A. E. (1996). One hundred years of forgetting: A quantitative description of retention. Psychological Review, 103, 734-760. doi:10.1037/0033-295X.103.4.734
Simen, P., Balci, F., Cohen, J. D., \& Holmes, P. (2011). A model of interval timing by neural integration. Journal of Neuroscience, 31, 9238-9253.

Wearden, J. H., Philpott, K., \& Win, T. (1999). Speeding up and (... relatively ...) slowing down an internal clock in humans. Behavioural Processes, 46, 63-73. doi:10.1016/S0376-6357(99) 00004-2 